\title{
A Unique Case of Arterial Thrombosis and Recurrent CVA in ICU: Unfathomable Presentation of an Occult Malignancy
}

\author{
Rajavardhan Rangappa ${ }^{1}$, Rajesh M Shetty ${ }^{2}$, Maria Denzil ${ }^{3}$, Pradeep E Haranahalli ${ }^{4}$, Soumik Susmita ${ }^{5}$, Sheetal Chaurasia ${ }^{6}$
}

\begin{abstract}
Introduction: Critically ill patients may present with prothrombotic manifestations. Carcinoma cervix with prothrombotic manifestations are not common. Arterial thrombosis in such cases is very rare. We present a case of carcinoma cervix which posed a diagnostic dilemma and difficulty in localizing primary. This patient also had recurrent strokes and cardiac metastasis with metastatic arterial thrombosis.

Case description: A 34-year-old lady presented with a history of acute lower limb ischemia and recurrent strokes. Transthoracic echocardiography showed valvular vegetations. Prothrombotic and infective endocarditis workup were negative. Histopathological examination (HPE) of clot showed metastatic squamous cells. Contrast CT of chest and abdomen only showed mediastinal lymphadenopathy. Endobronchial ultrasound (EBUS) with mediastinal lymph node biopsy showed metastatic squamous cells. As the patient gave a history of hysterectomy, Pap smear from the vault was sent, which was suggestive of high grade squamous intraepithelial neoplasia. Palliative chemotherapy was started. The patient made a good recovery and was discharged home in a stable condition.

Conclusion: Arterial thrombosis is an uncommon manifestation of occult malignancy. Carcinoma cervix usually does not metastasize to heart, brain, and arteries, which was the case in our patient. A high index of suspicion and systematic evaluation can clinch the diagnosis even when rare complications of malignancy are presented by critically ill patients.

Clinical significance: Any unprovoked thrombotic episodes should be extensively worked up for occult malignancies. We present a case demonstrating challenges faced by critical care physicians and benefits of methodical evaluation when confronted with unusual presentation of a malignancy.

Keywords: Brain, Cardiac, Malignancy, Metastasis, Secondaries, Thrombosis, Vault malignancy.

Indian Journal of Critical Care Medicine (2020): 10.5005/jp-journals-10071-23481
\end{abstract}

\section{BACKGROUND}

Critically ill patients may present with prothrombotic manifestations. Though prothrombotic manifestations are one of the most common presentations of malignancies, other differential diagnoses need to be considered. Usually patients present with venous thrombosis and rarely arterial thrombosis. Pathogenesis is multifactorial. Carcinoma cervix generally presents with distal metastasis to the lung, lymph nodes, and abdomen. Distant metastasis indicates the aggressive nature of the tumor and poor prognosis. Prothrombotic manifestations in carcinoma cervix are not uncommon, but its manifestation with arterial thrombosis is very rare. We present a case of carcinoma cervix which posed a diagnostic dilemma and difficulty in localizing primary and had recurrent strokes, cardiac metastasis with metastatic arterial thrombosis.

\section{Case Description}

A 34-year-old female with a history of cerebrovascular accident 1 month back with left-sided hemiparesis presented with a history of severe right leg pain for 8-10 days, progressive blackish discoloration of the right lower limb for 3 days. Echocardiographic evaluation showed floating, pedunculated, soft thrombus in the cardiac chambers, attached to left atrial appendage, mitral apparatus, right atrial cavity, and at right ventricle apex (Fig. 1). CT angiography showed a common filling defect within the femoral bifurcations extending to the superficial femoral artery. The proximal and mid-superficial femoral artery showed absent filling. Reconstitution of flow seen in the distal femoral artery.
${ }^{1-3}$ Department of Critical Care Medicine, Manipal Hospital Whitefield,
Bengaluru, Karnataka, India
${ }^{4}$ Department of Cardiology, Manipal Hospital Whitefield, Bengaluru,
Karnataka, India
${ }^{5}$ Department of Pathology, Manipal Hospital Whitefield, Bengaluru,
Karnataka, India
${ }^{6}$ Department of Pulmonology, Manipal Hospital Whitefield, Bengaluru, Karnataka, India

Corresponding Author: Rajavardhan Rangappa, Department of Critical Care Medicine, Manipal Hospital Whitefield, Bengaluru, Karnataka, India, Phone: +91 8589969639, e-mail: rajavardhanr99@ gmail.com

How to cite this article: Rangappa R, Shetty RM, Denzil M, Haranahalli PE, Susmita S, Chaurasia S. A Unique Case of Arterial Thrombosis and Recurrent CVA in ICU: Unfathomable Presentation of an Occult Malignancy. Indian J Crit Care Med 2020;24(7):585-588.

Source of support: Nil

Conflict of interest: None

Popliteal artery was not opacified suggestive of occlusion (Fig. 2). The patient underwent emergency right sided superficial femoral arterial thromboembolectomy (for SFA long segment thrombosis) and below knee amputation. Infective endocarditis with distal embolization was suspected, and the patient was started on empirical antibiotics. Post procedure she was started on heparin infusion along with antiplatelets.

(0) The Author(s). 2020 Open Access This article is distributed under the terms of the Creative Commons Attribution 4.0 International License (https://creativecommons. org/licenses/by-nc/4.0/), which permits unrestricted use, distribution, and non-commercial reproduction in any medium, provided you give appropriate credit to the original author(s) and the source, provide a link to the Creative Commons license, and indicate if changes were made. The Creative Commons Public Domain Dedication waiver (http://creativecommons.org/publicdomain/zero/1.0/) applies to the data made available in this article, unless otherwise stated. 


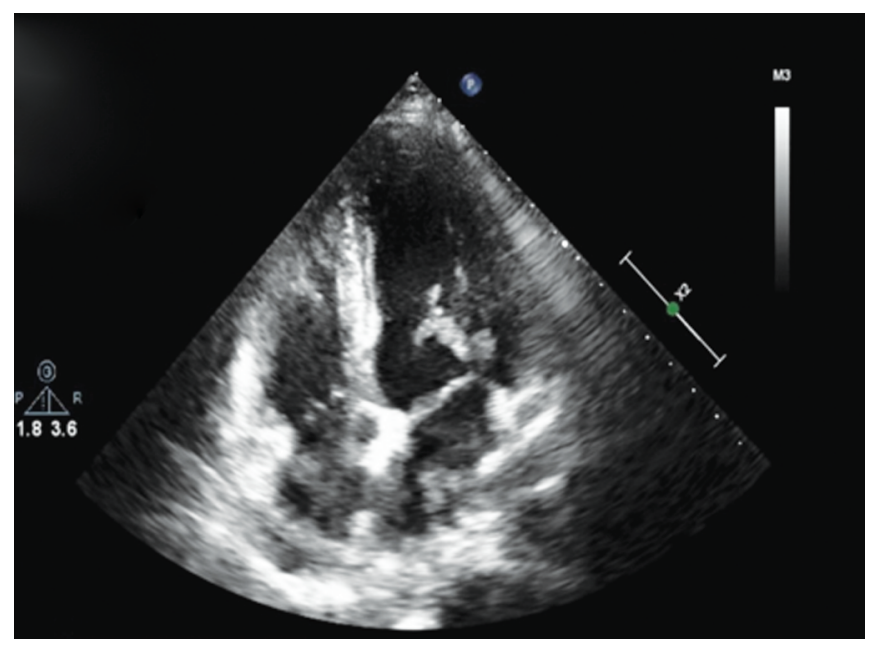

Fig. 1: Transthoracic echo 4-chamber and short axis view showing pedunculated thrombus attached to cardiac chambers

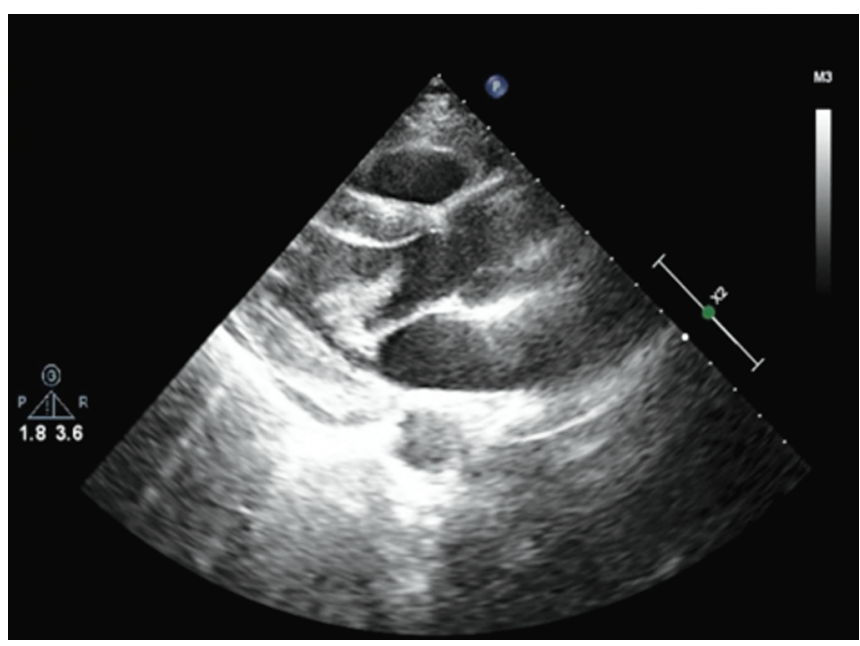

Fig. 2: Transthoracic echo 4-chamber and short axis view showing pedunculated thrombus attached to cardiac chambers

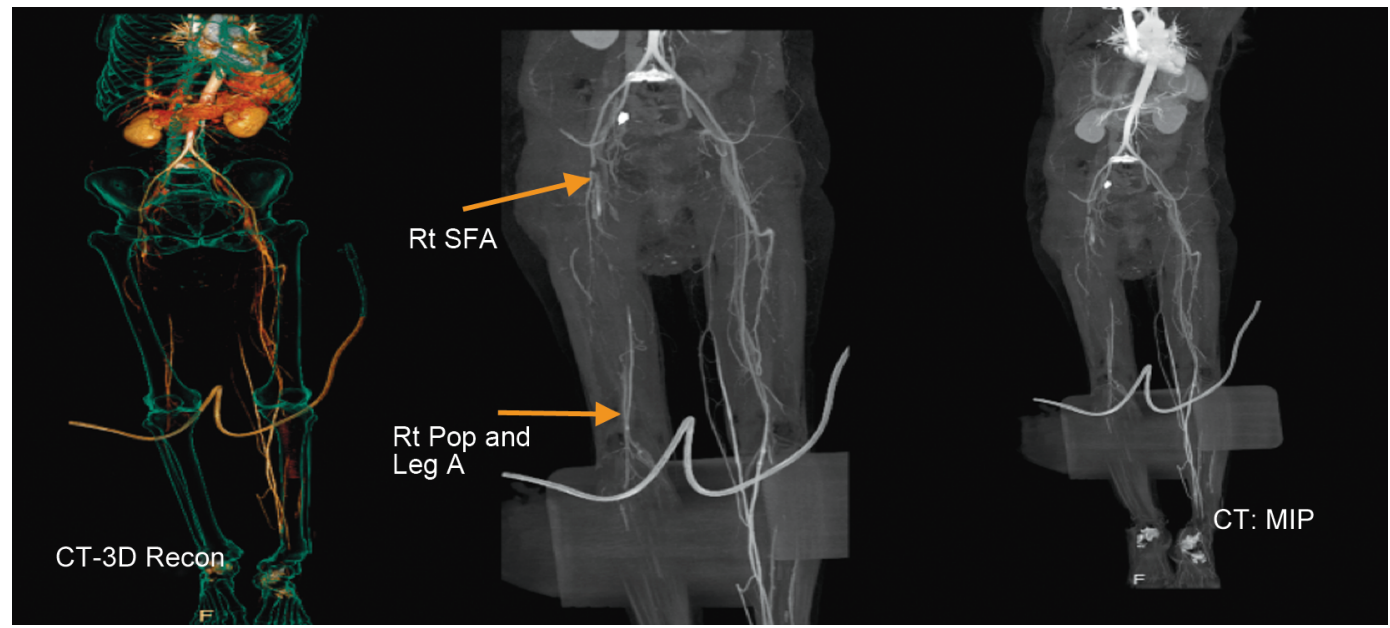

Fig. 3: Common femoral artery showing filling defect seen within the femoral bifurcations extending to the superficial femoral artery. The proximal and mid superficial femoral artery shows absent filling. Reconstitution of flow seen in the distal femoral artery

Multiple sets of blood cultures were sent, which were reported as negative. Prothrombotic workup was sent including anti-nuclear acid test, antiphospholipid antibody workup, and homocysteine levels. All the tests were negative. On the seventh day of hospital stay, the patient was noted to have newly onset dysarthria and delirium. MRI brain showed new acute infarct in the left parietal region. Hyperacute left middle cerebral artery (MCA)/posterior cerebral artery (PCA) borderline infarct. Old MCA infarct right sided with embolic infarcts in right MCA territory; focal thrombosis in right internal carotid artery (ICA) and absence of flow in the distal ICA and wall thickening in right common carotid artery; and focal irregularity in right PCA. CT chest and abdomen showed left upper and lower lobe pulmonary veins thrombus. Mediastinal lymphadenopathy and few lung nodules were present. A few airspace opacities and patchy consolidation were seen in the left upper lobe. Both kidneys (right more than the left) showed segmental wedge-shaped nonenhancing area suggestive of infarcts. Main renal arteries were patent. Other findings included right internal iliac artery occlusion, right common femoral artery occlusion extending to the superficial femoral artery, nonopacified popliteal artery, suggesting occlusion and few collaterals in the thigh.

Histopathological examination (HPE) of the clot showed metastatic squamous cells (Figs 3 and 4). EBUS with mediastinal lymph node biopsy showed metastatic squamous cells (Fig. 5). The family reported a history of hysterectomy 1 year back with no histopathology reports available. Hence, it was decided to send Pap smear from the vault, which was suggestive of high grade squamous intra epithelial neoplasia (Figs 6 and 7). Oncology opinion was taken and a diagnosis of metastatic squamous cell carcinoma with vaginal vault as most likely primary with endocardial secondaries was made and initiated on palliative chemotherapy with paclitaxel and carboplatin. Bronchioalveolar lavage was done and the culture showed Pseudomonas aeruginosa. Antibiotic was adjusted according to the culture and sensitivity report. Patient was gradually bridged with warfarin and discharged home.

\section{Discussion}

Malignancies are associated with thrombotic manifestations. Pathogenesis can be multifactorial, and the presentation of 


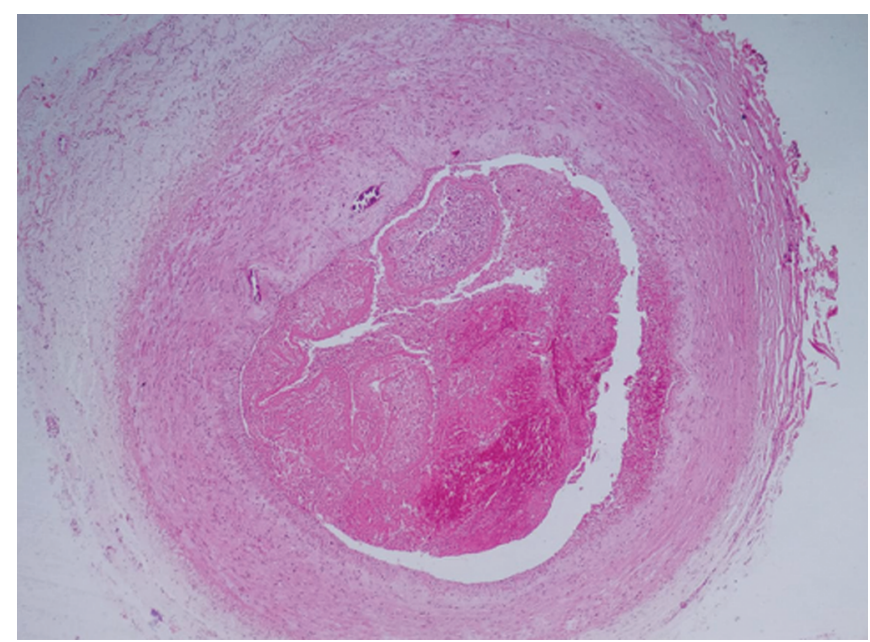

Fig. 4: Arterial lumen with tumor

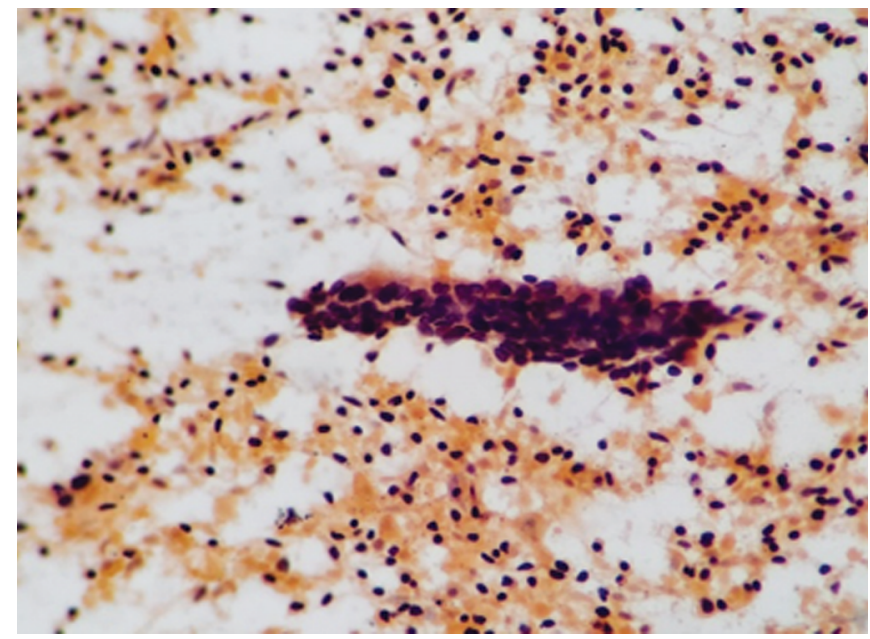

Fig. 6: HPE of BAL with atypical squamous cell clusters

thrombotic manifestations might be related to prothrombotic condition or mechanical obstruction causing thrombosis. Prothrombotic process might be related to neoplastic or paraneoplastic process. Hypercoagulative condition may be related to changes in the level of factor VII, protein C and S, tissue factor, cancer procoagulant levels, thrombocytosis, or increased fibrionogen levels. Venous thromboembolism has been widely described. Arterial thrombosis and cardiac metastasis are relatively rare compared to venous thrombosis., ${ }^{1,2}$ There are isolated case reports and few studies. In a study by El Sakka et al., patients presenting with critical limb ischemia were analyzed: out of 192 patients presenting with critical limb ischemia, 22 were found to be associated with malignancy. ${ }^{3} \mathrm{~A}$ high index of suspicion is required, and occult malignancy should be considered in the evaluation of patients presenting with arterial thrombosis.

Carcinoma cervix is the most common malignancy in Indian woman, with an incidence of 19-44 per 100,000 women. Carcinoma cervix spreads locally and exhibits a low degree of distant metastasis. Metastasis to distant sites is very unusual. Metastasis in carcinoma cervix can occur via lymphatic spread or hematogenous spread. Hematogenous spread in carcinoma cervix can occur via venous plexus or the paracervical veins.

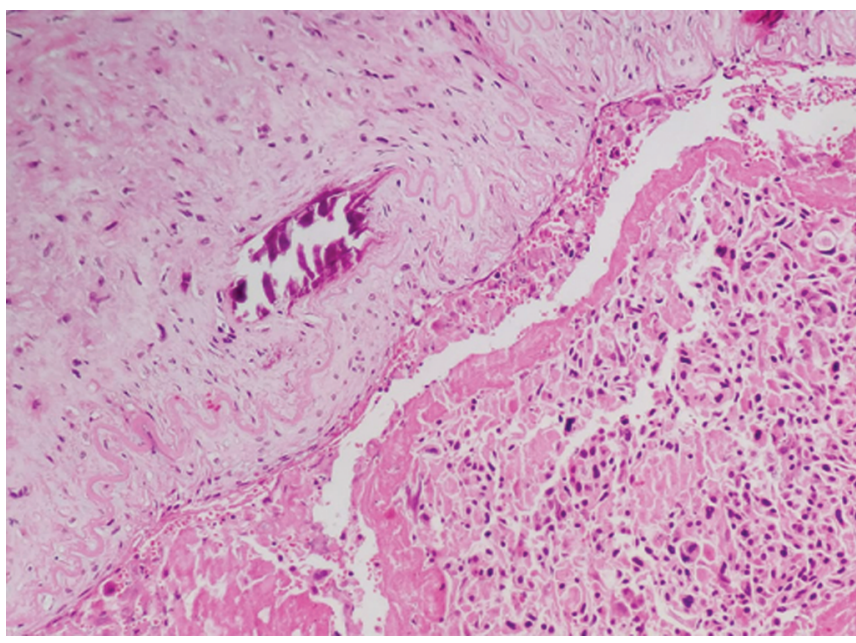

Fig. 5: Arterial lumen with blood vessel invasion

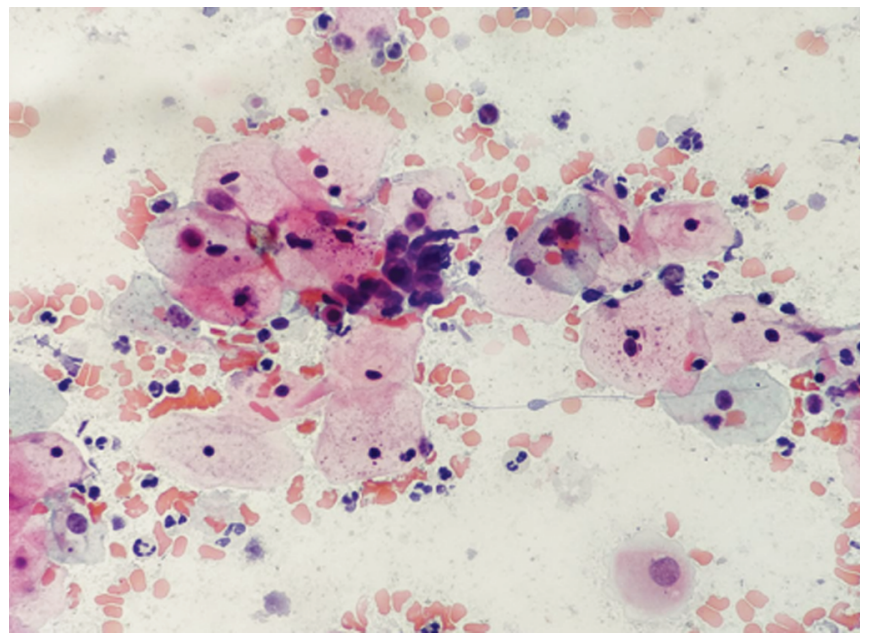

Fig. 7: Vault smear with atypical squamous cells

The common site of distant metastasis includes lungs, liver, bones, and supraclavicular nodes. The rare sites include heart, brain, and muscles. ${ }^{4}$ Most recurrences occur within 2 years of diagnosis, and the prognosis is poor, with most patients dying as a result of uncontrolled disease.

Vault carcinoma or recurrence as the primary is very rare; there are only few reported cases. Most of them are secondary to primary in cervix or vagina; some of them secondary to carcinoma ovary/ breast and only only paper reported first-time presentation in the vault as primary. ${ }^{5,6}$

\section{ConClusion}

Arterial thrombosis is an uncommon manifestation of occult malignancy, especially carcinoma cervix. Carcinoma cervix usually does not metastasize to heart, brain, and arteries, which was the case in our patient. A high index of suspicion and systematic evaluation can clinch the diagnosis even when rare complications of malignancy are presented by critically ill patients. Our case was unique in the fact that it was vault recurrence as the primary from most likely carcinoma cervix and endocardial secondaries with distant metastatic embolization in the heart, lungs, and brain. 


\section{Clinical Significance}

Any unprovoked thrombotic episodes should be extensively worked up for occult malignancies. We present a case demonstrating challenges faced by critical care physicians and benefits of methodical evaluation when confronted with unusual presentation of a malignancy.

\section{References}

1. Purkayastha A, Singh S, Bisht N, Mishra P, Husain A. Squamous cell carcinoma of vaginal vault with liver, adrenal and brain metastases: report of an uncommon presentation. ARC J Can Sci 2018;4(2): $1-6$.
2. El Sakka K, Gambhir R, Halawa M, Chong P, Rashid H. Association of malignant disease with critical leg ischaemia. Br J Surg 2005;92(12):1498-1501. DOI: 10.1002/bjs.5125.

3. Javid M, Magee T, Galland R. Arterial thrombosis associated with malignant disease. Eur J Vasc Endovasc Surg 2008;35(1):84-87. DOI: 10.1016/j.ejvs.2007.08.014.

4. Dunster M, Bennett D. Primary carcinoma of the vaginal vault following total hysterectomy for non-malignant conditions. Bjog: An Int J Obstet Gynaecol 1953;60(1):126-128. DOI: 10.1111/j.14710528.1953.tb06426.x.

5. Bussani R, De-Giorgio F, Abbate A, Silvestri F. Cardiac metastases. J Clin Pathol 2007;60(1):27-34. DOI: 10.1136/jcp.2005.035105.

6. Shrivastava S, Barmon D, Deka P, Kataki A. Vaginal vault carcinoma as second primary in a treated case of ovarian cancer. J Midlife Health 2012;3(2):106. DOI: 10.4103/0976-7800.104476. 